\title{
Factors predicting opioid dependence in patients undergoing surgery for degenerative spondylolisthesis: analysis from the MarketScan databases
}

\author{
Mayur Sharma, MD, MCh, ${ }^{1}$ Beatrice Ugiliweneza, PhD, MSPH, ${ }^{1}$ Zaid Aljuboori, MD, ${ }^{1}$ \\ Miriam A. Nuño, PhD, ${ }^{2}$ Doniel Drazin, MD, ${ }^{3}$ and Maxwell Boakye, MD, MPH, MBA ${ }^{1}$ \\ 1Department of Neurosurgery, University of Louisville, Louisville, Kentucky; ${ }^{2}$ Department of Public Health Sciences, Division \\ of Biostatistics, University of California Davis, Davis, California; and '3Department of Neurosurgery, Swedish Medical Center, \\ Seattle, Washington
}

OBJECTIVE The opioid crisis is identified as a national emergency and epidemic in the United States. The aim of this study was to identify risk factors associated with opioid dependence in patients undergoing surgery for degenerative spondylolisthesis (DS).

METHODS The authors queried MarketScan databases to investigate the factors affecting postsurgery opioid use in patients with DS between 2000 and 2012. The outcome of interest was opioid dependence, which was defined as continued opioid use, > 10 opioid prescriptions, or diagnosis of or prescription for opioid dependence disorder in the period of 1 year before or 3-15 months after the procedure. Comparisons of outcomes were performed using nonparametric 2-group tests and generalized regression models.

RESULTS A cohort of 10,708 patients was identified from the database. The median patient age was 61 years (interquartile range $54-69$ years), and $65.1 \%$ were female $(n=6975)$. A majority of patients had decompression with fusion $(n=10,068 ; 94 \%)$ and underwent multilevel procedures $(n=8123 ; 75.9 \%)$. Of 10,708 patients, $14.85 \%(n=1591)$ were identified as having opioid dependence within 12 months prior to the index surgical procedure and $9.90 \%(n=1060)$ were identified as having opioid dependence within 3-15 months after the procedure. Of all the variables, prior opioid dependence (OR 16.29, 95\% Cl 14.10-18.81, p < 0.001) and younger age (1-year increase in age: OR 0.972, 95\% Cl 0.963-0.980, $p<0.001$ ) were independent predictors of opioid dependence following surgery for DS. The use of fusion was not associated with opioid dependence following the procedure $(p=0.8396)$. Following surgery for DS, patients were more likely to become opioid independent than they were to become opioid dependent $(8.54 \%$ vs $3.58 \%, p<$ 0.001).

CONCLUSIONS The majority of patients underwent fusion for DS. Surgical decompression with fusion was not associated with increased risk of postsurgery opioid dependence in patients with DS. Overall, opioid dependence was reduced by $4.96 \%$ after surgery for DS. Prior opioid dependence is associated with increased risk and increasing age is associated with decreased risk of opioid dependence following surgery for DS.

https://thejns.org/doi/abs/10.3171/2018.1.SPINE171258

KEYWORDS lumbar decompression; fusion; opioid; degenerative spondylolisthesis

$\mathrm{T}$ HE opioid crisis is identified as a national emergency and epidemic in the United States. ${ }^{1}$ More than 12 million people misuse prescription opioids, and prescription opioid overdose is a leading cause of drug overdose-related deaths (60.9\%) in this country. ${ }^{23}$ Given the extent of this health care problem, it is imperative to understand the risk factors predictive of long-term opioid dependence and to take necessary steps in curtailing this opioid epidemic.

The aim of this study was to identify risk factors associated with opioid dependence in patients undergoing surgery for degenerative spondylolisthesis (DS), which

ABBREVIATIONS $\mathrm{Cl}=$ confidence interval; $\mathrm{CPT}-4=$ Current Procedural Terminology, Fourth Edition; $\mathrm{DS}=$ degenerative spondylolisthesis; ICD-9-CM = International Classification of Diseases, Ninth Revision, Clinical Modification; IQR = interquartile range; OR = odds ratio; RR = relative risk.

SUBMITTED November 20, 2017. ACCEPTED January 11, 2018.

INCLUDE WHEN CITING Published online June 19, 2018; DOI: 10.3171/2018.1.SPINE171258. 
is a frequent cause of spinal stenosis in elderly patients (male patients mean age 68 years and females mean age 71 years), affecting primarily Caucasian women (5 times higher than men) with an estimated prevalence of approximately 6\%.13 The Spine Patient Outcomes Research Trial (SPORT) reported superior outcomes at the 2-year follow-up for surgical compared to nonsurgical management of patients with DS. ${ }^{29}$ However, there is a lack of consensus regarding what type of surgical procedure provides the most effective treatment for DS, and commonly used procedures include laminectomy or decompression with or without fusion. In a recent randomized controlled trial, patients with grade 1 DS treated with laminectomy and posterolateral fusion had superior physical health-related quality of life (based on responses to the SF-36) at the 4-year follow-up compared to patients treated with laminectomy alone. ${ }^{12}$ Interestingly, another randomized Swedish study showed no significant differences in outcomes at the 2- and 5-year follow-up after either decompression alone or decompression with fusion in patients with spinal stenosis with or without spondylolisthesis. ${ }^{11}$ Despite this controversy regarding choice of treatment, a majority of patients with DS in the United States are managed with decompression combined with fusion. ${ }^{14,19}$ Opioids are commonly prescribed following common spine surgeries (discectomy, decompression, lumbar posterolateral arthrodesis, or lumbar interbody arthrodesis) and this use of prescription opioids may lead to prolonged use in only a minority of patients $(0.1 \%$ at 6 months $),{ }^{24}$ in particular those with prior opioid dependence or preexisting mental health disorders such as depression., ${ }^{4,7,20,24}$ A recent study using a commercial insurance database showed that refusion lumbar spine surgeries were associated with increased risk and anterior lumbar fusion was associated with decreased risk of opioid dependence following lumbar fusion surgeries. ${ }^{6}$ The use of minimally invasive procedures has been shown to reduce the duration of postoperative narcotic use to half (median 2 weeks vs 4 weeks) and result in shorter hospital stays compared to open procedures for DS. ${ }^{2,20}$

Recently, there has been a surge in the use of national databases to query a variety of questions aiming to increase understanding of national trends and evaluate the efficacy of treatment modalities. , $^{3,8,18,25}$ In this study we used information from available databases to investigate factors that may increase the risk of opioid dependence in patients undergoing surgical treatment for DS. To the best of our knowledge, this is the first study focusing on the risk factors associated with opioid dependence in this patient population with DS, which is a common clinical problem associated with significant health care utilization. ${ }^{26}$

\section{Methods \\ Data Sources}

We used the MarketScan Commercial claims and encounters, Medicare Supplemental, and Multi-State Medicaid databases to investigate the impact of surgery on opioid use in patients with DS (https://truvenhealth.com/ markets/life-sciences/products/data-tools/marketscan- databases). The MarketScan databases capture patientlevel data on clinical utilization, insurance enrollment, and costs linked with detailed patient, provider, and facility information. The databases include longitudinal data grouped into multiple files, including inpatient, outpatient, and prescription medication, and use an encrypted ID for each individual, which serves as a unique identifier and a linkage variable across different files..$^{10}$ The information in the MarketScan databases is obtained from paid patient health insurance claims from approximately 100 data sources and is available for purchase from Truven Health Analytics-IBM Watson Health. Included are data for covered individuals and their dependents. Patient information enters the databases when patients enroll in health insurance coverage and exits when that enrollment ends; patient interactions with the health care system are captured in the MarketScan data through their claims. These data have been used extensively in comparative effectiveness research in the medical field since 1990.

\section{Patient Selection and Inclusion/Exclusion Criteria}

From the inpatient admission tables, we extracted data on patients with a primary diagnosis of acquired lumbar spondylolisthesis (ICD-9-CM [International Classification of Diseases, Ninth Revision, Clinical Modification] code 738.4) and concurrent procedures of either a decompression-only procedure or decompression with fusion. Another inclusion criterion was age 18 years or older at the time of index hospitalization. Exclusion criteria were pregnancy, intraspinal abscess, osteomyelitis, fracture, vertebral dislocation, accidents, or pelvic instrumentation during the index procedure. Also, data on patients lacking medical records for the period of 1 year presurgery to 2 years postsurgery were excluded (Fig. 1).

\section{Calculation of Follow-Up Time}

Preoperative follow-up time was calculated as the time difference between the start of enrollment and the index hospitalization admission. If the start enrollment time was not available because the enrollment started prior to our data window, the beginning of our data window-January 1,2000-was used. Similarly, postoperative follow-up time was calculated as the difference between the index hospitalization discharge date and the end of enrollment. If the end of enrollment fell outside of the data period, we used December 31, 2012, which was the last date of our data window.

\section{Explanatory Variables}

We used baseline characteristics at the index hospitalization as the explanatory variables. These were age, sex, year of index hospitalization, preoperative follow-up months, postoperative follow-up months, insurance type (commercial, Medicaid, or Medicare), comorbidities, instrumentation, and number of spinal levels. Comorbidities were measured with the Elixhauser comorbidity score, ${ }^{9}$ computed using an adaptation to ICD-9-CM codes developed by Quan et al. ${ }^{21}$ The Elixhauser score is an index that quantifies the burden of comorbidities abstracted from medical or claims records using ICD codes. A total 
of 30 comorbidities are checked as being present or not (present $=1$, absent $=0$ ) and cumulated to provide a comorbidity score. To check whether instrumentation was used, we screened the CPT-4 (Current Procedural Terminology, Fourth Edition) procedure codes 22840-22847. Cases were classified as fusion involving multiple spinal levels ( $\geq 2$ levels) if the following codes were found: ICD9 codes 81.62, 81.63, 81.64, and/or CPT-4 codes 22585, $22632,22614,63035,63044,63048,63057,63103$, and 22842-22847.

\section{Postoperative Outcome Variables}

The current study aimed to identify factors predictive of postsurgery opioid dependence.

\section{Opioid Use}

Our interest was to evaluate opioid dependence postsurgery (in the period from 3 to 15 months postdischarge). However, we also looked at use prior to surgery (within 12 months before admission) in order to identify new dependence. Patients were classified as having opioid dependence if they had a diagnosis of opioid use disorder (ICD9 305.50, 305.55, 305.52, 304.00, 304.01, 304.02, 304.70, 304.71 , or 304.72$),{ }^{6}$ obtained a prescription for a medication used to treat opioid use disorder (buprenorphine or naltrexone), were counseled about psychological and pharmacological treatment options for opioid addiction (CPT$44306 \mathrm{~F}),{ }^{6}$ or had at least 10 prescriptions ${ }^{7,17,22,28}$ of opioid drugs (buprenorphine, butorphanol, codeine phosphate, dihydrocodeine, fentanyl citrate, hydrocodone, hydromorphone, levorphanol, meperidine, methadone, morphine sulfate, nalbuphine, opium alkaloids, oxycodone, oxymorphone, pentazocine, propoxyphene, tapentadol, tramadol, or naltrexone $)^{16}$ in the look-up period (12 months prior to admission for presurgery dependence and 3-15 months ${ }^{5,28}$ after discharge for postsurgery dependence).

\section{Statistical Analysis}

We summarized continuous variables using means and standard deviations, medians and interquartile ranges, and full ranges (minimum to maximum). Categorical variables were summarized using counts and percentages.

To compare the patient characteristics and outcomes among different procedure groups, we used linear regression on logged values for continuous variables and logistic regression for categorical variables. ${ }^{15}$ The models included the procedure group as well as age, sex, insurance type (commercial, Medicaid, or Medicare), Elixhauser comorbidity score, presence of instrumentation, and whether multiple levels were involved as independent variables. We used the software SAS 9.4 (SAS Institute, Inc.) for data preparation and data analysis. ${ }^{27}$

\section{Results \\ Patient Demographics}

A total of 10,708 patients with a diagnosis of degenerative spondylolisthesis (DS) who underwent a surgical procedure were identified from the MarketScan databases.

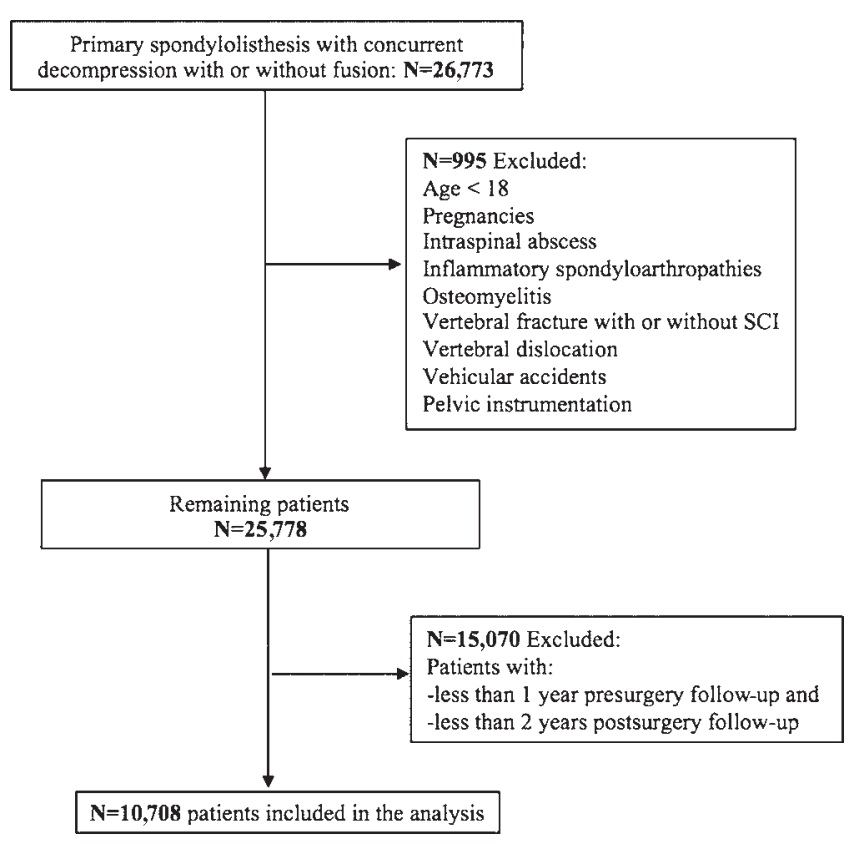

FIG. 1. Flowchart showing the inclusion and exclusion criteria used for extracting data from the MarketScan databases.

The median patient age was 61 years (interquartile range [IQR] 54-69 years). A majority of the patients were female $(\mathrm{n}=6975,65.1 \%)$. Commercial insurance was the most common insurance type $(61.4 \%, \mathrm{n}=6571)$, followed by Medicare $(35 \%, \mathrm{n}=3746)$. Less than half of the patients $(46 \%, n=4954)$ had an Elixhauser comorbidity score of 0, and a minority $(8.07 \%, \mathrm{n}=864)$ had a comorbidity index of $3+$. Most of the patients underwent decompression with fusion ( $\mathrm{n}=10,068,94 \%)$, and approximately three-quarters had multilevel procedures $(n=8,123,75.9 \%)$.

Demographic and clinical characteristics of the patients who underwent surgical treatment for DS are shown in Table 1.

\section{Opioid Dependence}

The incidence of postsurgery opioid dependence gradually increased from $11.67 \%$ (2001) to $16.12 \%$ (2011) among patients who were opioid dependent prior to the surgical procedure and from $6.67 \%$ (2001) to $10.81 \%$ (2011) among patients who were opioid independent prior to the surgery (Fig. 2). A total of 1591 patients (14.85\%) were identified to have opioid dependence within 12 months prior to the index surgical procedure and $1060(9.90 \%)$ were identified to have opioid dependence within 3-15 months after the procedure. A majority of patients $(90.1 \%, \mathrm{n}=9648)$ were opioid independent following the surgical procedure. Following surgery for DS, opioid-dependent patients were 2 times more likely to become opioid independent than opioid-independent patients were likely to become opioid dependent $(8.54 \%$ vs $3.58 \%$, odds ratio [OR] $2.39,95 \%$ confidence interval [CI] 2.12-2.69, p < 0.0001; Fig. 3).

\section{Factors Predicting Opioid Dependence Following Surgery for DS}

Comparison of demographic and clinical characteris- 
TABLE 1. Summary of patient demographic and clinical characteristics

\begin{tabular}{|c|c|c|c|c|}
\hline \multirow[b]{2}{*}{ Variable } & \multirow{2}{*}{$\begin{array}{l}\text { Combined Cohort, } \\
\qquad n=10,708\end{array}$} & \multicolumn{2}{|c|}{ Postsurgery Opioid Dependence } & \multirow[b]{2}{*}{$p$ Value } \\
\hline & & No, $n=9648(90.1 \%)$ & Yes, $n=1060(9.90 \%)$ & \\
\hline Age, yrs & & & & $<0.0001$ \\
\hline Mean (SD) & $60.7(11.9)$ & $61.1(11.8)$ & $57.0(12.1)$ & \\
\hline Median (IQR) & $61.0(54.0-69.0)$ & $61.0(54.0-70.0)$ & $57.0(49.0-65.0)$ & \\
\hline Range, min-max & $18.0-95.0$ & $18.0-95.0$ & $20.0-92.0$ & \\
\hline Sex: female, $n(\%)$ & $6975(65.1 \%)$ & $6244(64.7 \%)$ & $731(69.0 \%)$ & 0.0059 \\
\hline Insurance, $\mathrm{n}(\%)$ & & & & $<0.0001$ \\
\hline Commercial & $6571(61.4 \%)$ & $5854(60.7 \%)$ & $717(67.6 \%)$ & \\
\hline Medicaid & $391(3.65 \%)$ & $322(3.34 \%)$ & $69(6.51 \%)$ & \\
\hline Medicare & $3746(35.0 \%)$ & $3472(36.0 \%)$ & $274(25.8 \%)$ & \\
\hline Elixhauser index, n (\%) & & & & 0.0825 \\
\hline 0 & $4954(46.3 \%)$ & $4441(46.0 \%)$ & $513(48.4 \%)$ & \\
\hline 1 & $3386(31.6 \%)$ & $3064(31.8 \%)$ & $322(30.4 \%)$ & \\
\hline 2 & $1504(14.1 \%)$ & $1376(14.3 \%)$ & $128(12.1 \%)$ & \\
\hline $3+$ & $864(8.07 \%)$ & $767(7.95 \%)$ & $97(9.15 \%)$ & \\
\hline Surgery: decompression, $\mathrm{n}(\%)$ & & & & 0.6022 \\
\hline w/o fusion & $640(5.98 \%)$ & $597(6.19 \%)$ & $43(4.06 \%)$ & \\
\hline w/ fusion & $10,068(94.0 \%)$ & $9051(93.8 \%)$ & $1017(95.9 \%)$ & \\
\hline Multilevel surgery, n (\%) & $8123(75.9 \%)$ & $7312(75.8 \%)$ & $811(76.5 \%)$ & \\
\hline
\end{tabular}

Max = maximum; $\min =$ minimum; $\mathrm{SD}=$ standard deviation.

Boldface type indicates statistical significance.

tics of patients with and without opioid dependence following surgery showed that younger age, female sex, and having commercial insurance were associated with opioid dependence following surgery (median age 57 vs 61 years, $\mathrm{p}<0.0001$; female sex $69 \%$ vs $64.7 \%, \mathrm{p}=0.0059$; and commercial insurance $67.6 \%$ vs $60.7 \%, \mathrm{p}<0.0001$ ). Comorbidity index and type of surgical procedure had no association with postsurgery opioid dependence on univariable analysis $(p>0.05$, Table 1$)$.

Of all the variables, prior opioid dependence (OR 16.29,
95\% CI 14.10-18.81, p < 0.001) and younger age (1-year increase in age: OR $0.972,95 \%$ CI $0.963-0.980, p<0.001$ ) were independent predictors of opioid dependence following surgery for DS. Type of surgical procedure (decompression alone or decompression with fusion) showed an association with postsurgery opioid dependence on univariable analysis $(6.72 \%$ vs $10.10 \%, p=0.0009 ;$ Fig. 4 and Table 2). Also, $41.9 \%$ of patients who were not opioid dependent before surgery became opioid dependent following decompression without fusion compared to $35.90 \%$ of

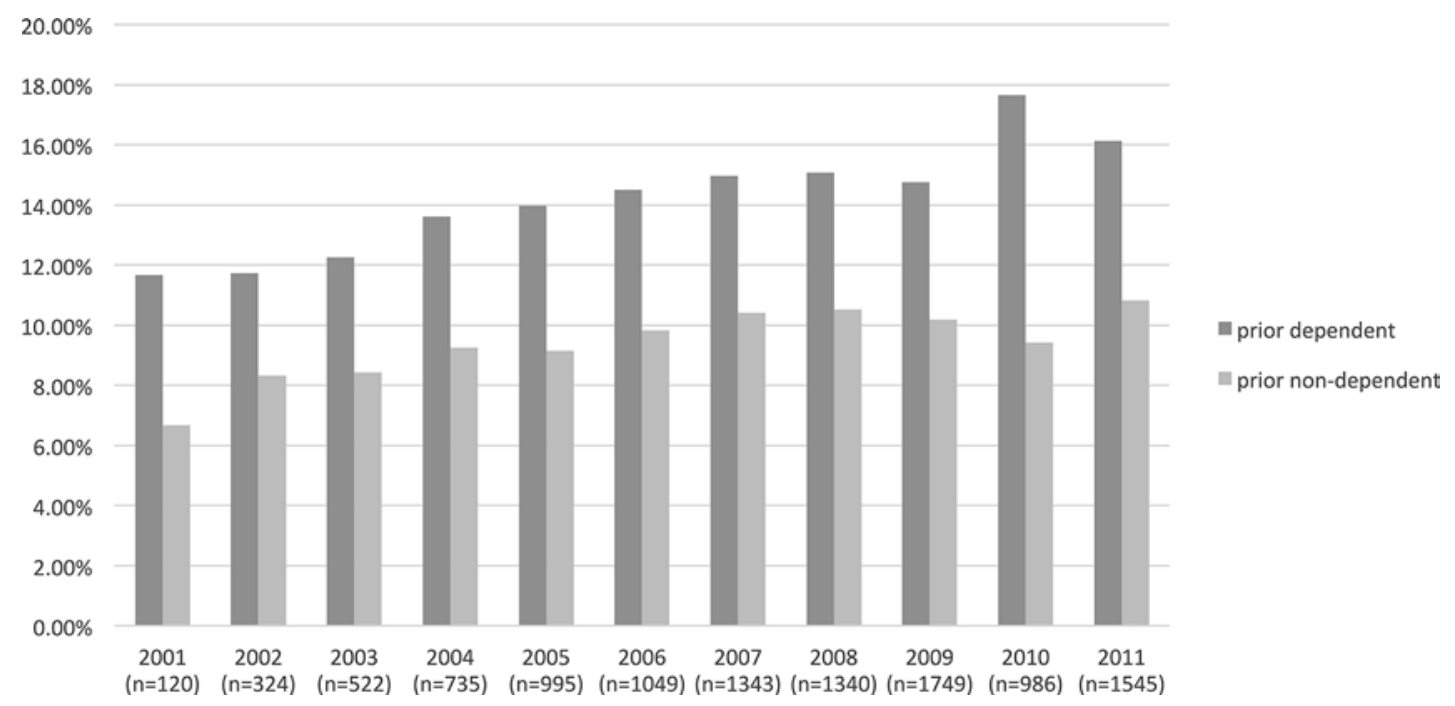

FIG. 2. Bar graph showing the temporal trends (2001-2011) in the incidence of postoperative opioid dependence among patients who were previously opioid dependent vs those who were not previously opioid dependent following surgery for DS. 
Total $\mathrm{n}=10,708$

Presurgery dependent

Postsurgery dependent

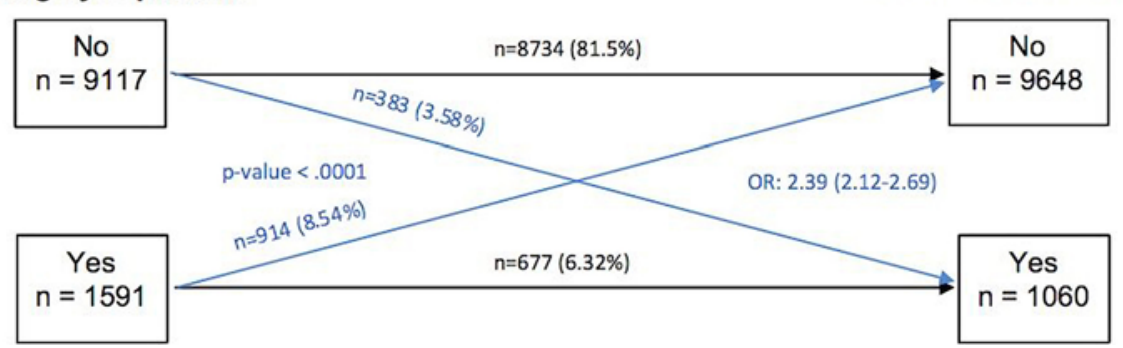

FIG. 3. Flowchart showing changes in opioid dependence among patients who underwent surgery for DS. Figure is available in color online only.

patients who underwent decompression with fusion $(\mathrm{p}>$ 0.05 , Fig. 5). However, on adjusted multivariable analysis, the use of fusion was not associated with opioid dependence following the procedure (OR 1.038, 95\% CI 0.726$1.483, \mathrm{p}=0.8396$ ). Elixhauser comorbidity score, type of insurance, and number of levels treated had no significant association with opioid dependence following surgery for DS (Table 3).

\section{Discussion}

Using the MarketScan databases, we found that $90.1 \%$ of all patients were opioid independent following DS surgery compared to $85.15 \%$ prior to surgery. Patients were twice as likely to become opioid independent after surgery as they were to become opioid dependent (OR 2.39, 95\% CI $2.12-2.69, \mathrm{p}<0.001)$. Prior opioid dependence and young age were independent predictors of opioid dependence following surgery for DS. Type of surgical procedure had no impact on postsurgery opioid dependence.

In our study, $14.85 \%$ of patients were reported to be opioid dependent 1 year before surgery, and $9.90 \%$ were opi- oid dependent 3-15 months after surgery. A recent study based on commercial insurance claims reported continued narcotic utilization in $62 \%$ of patients more than 1 year following lumbar fusion surgery. ${ }^{18}$ However, the number of patients with opioid dependence prior to the surgery was not reported in that study. In contrast, we found that a majority of patients $(90.1 \%)$ were opioid independent after surgery for DS. Also, patients were twice as likely to become opioid independent following surgery for DS. In a recent study, Schoenfeld et al. ${ }^{24}$ used the TRICARE insurance claim database to investigate factors predictive of opioid dependence in patients $(\mathrm{n}=9991)$ undergoing spinal surgery for a variety of lumbar pathologies, including spondylolisthesis $(n=252)$ in opioid-naïve patients. In this study the authors reported that $1 \%$ of patients continued opioid use at 3 months and $0.1 \%$ continued use at 6 months postsurgery. They also reported that patients who underwent low-intensity surgical procedures, such as discectomy and decompression, were more likely to be opioid independent than those who underwent fusion procedures (HR 1.43 and 1.34, respectively). In our study we found that $3.58 \%$ of patients became opioid dependent after the

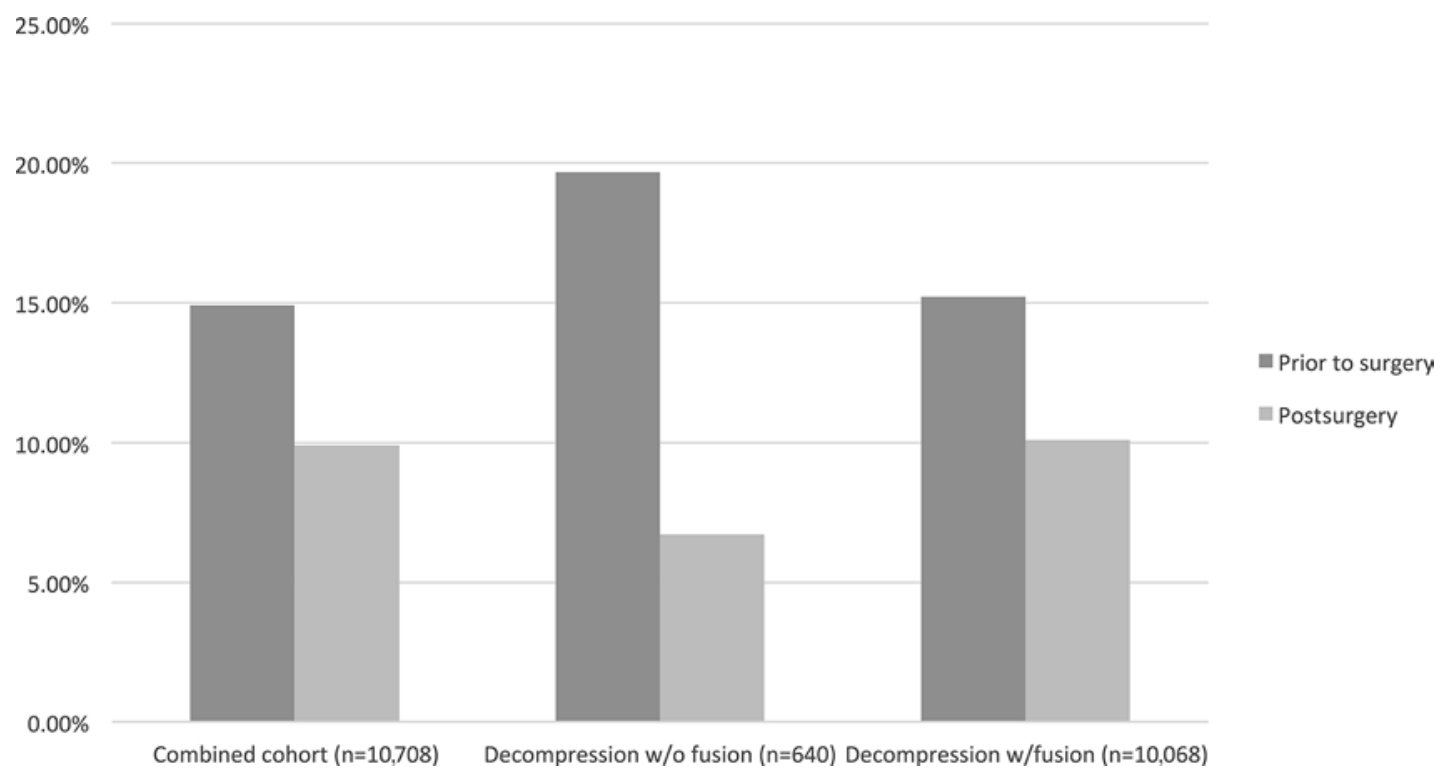

FIG. 4. Bar graph showing the rate of opioid dependence before and after surgery in the combined cohort, in patients who underwent decompression without fusion, and in patients who underwent decompression with fusion for DS. 
TABLE 2. Opioid dependence before and after surgery stratified by type of surgical procedure

\begin{tabular}{|c|c|c|c|c|}
\hline \multirow[b]{2}{*}{ Opioid Dependence Group } & \multirow[b]{2}{*}{$\begin{array}{l}\text { Combined Cohort, } \\
n=10,708(100 \%)\end{array}$} & \multicolumn{2}{|c|}{ Decompression } & \multirow[b]{2}{*}{ p Value } \\
\hline & & $\begin{array}{c}\text { w/o Fusion, } \\
n=640(5.98 \%)\end{array}$ & $\begin{array}{c}\text { w/ Fusion, } \\
n=10,068(94.02 \%)\end{array}$ & \\
\hline Previously nondependent pts who remained nondependent, $\mathrm{n}(\%)$ & $8734(81.6 \%)$ & $560(87.5 \%)$ & $8174(81.2 \%)$ & \multirow{4}{*}{0.0009} \\
\hline Previously nondependent pts who became dependent, n (\%) & $383(3.58 \%)$ & $18(2.81 \%)$ & $365(3.63 \%)$ & \\
\hline Previously dependent pts who became nondependent, $\mathrm{n}(\%)$ & $914(8.54 \%)$ & $37(5.78 \%)$ & $877(8.71 \%)$ & \\
\hline Previously dependent pts who remained dependent, $n(\%)$ & $677(6.32 \%)$ & $25(3.91 \%)$ & $652(6.48 \%)$ & \\
\hline
\end{tabular}

Pts = patients.

Boldface type indicates statistical significance.

surgical procedure for DS; $95.3 \%(n=365)$ of these patients had decompression with fusion, and $4.7 \%(\mathrm{n}=18)$ had only decompression. Therefore overall, only $0.16 \%$ of patients became opioid dependent following decompression only for DS, which is in concordance with the findings of the above-mentioned study. ${ }^{24}$ Schoenfeld et al. also identified depression as a factor associated with decreased likelihood of opioid independence following spinal surgery, which was also reported by Connolly et al. ${ }^{7}$ In contrast, we did not find any association between various comorbidities and opioid dependence in our study. This difference may be attributable to the highly selective patient population in our study - patients with a diagnosis of DS who underwent surgery treatment for that condition-compared to patients undergoing lumbar fusion procedures for a variety of spinal pathologies in the studies by Schoenfeld et al. ${ }^{24}$ and Connolly et al.

Another study based on a commercial insurance database reported that the duration of opioid use before surgery was significantly associated with an increased risk of long-term opioid dependence after lumbar fusion surgery. ${ }^{7}$ Patients using opioids for 1-22 days or more than 250 days prior to the surgery were 2.27 and 219 times, respectively, more likely to be opioid dependent after lumbar fusion surgery. This study also reported that anterior fusion was associated with decreased risk and $360^{\circ}$ fusion was associated with increased risk (compared to posterior fusion) of long-term opioid dependence following the procedure. Similarly, Armaghani et al. ${ }^{4}$ reported that young age, anxiety disorder, and increased preoperative opioid use were associated with increased opioid use during the immediate postoperative period, and major surgical procedure, revision procedure, anxiety, and increased preoperative opioid use were associated with increased opioid dependence at 12 months postsurgery. In concordance, we found that patients who were opioid dependent prior to the surgery were likely to remain dependent following the surgery (relative risk [RR] 16.29, $\mathrm{p}<0.0001$ ). However, type of surgical procedure had no impact on opioid dependence in our study. Also, we found that patients with increasing age were less likely to be opioid dependent following surgery for DS. This difference may be due to the different patient population in our study.

\section{Strengths and Limitations of the MarketScan Databases}

The advantages of using a nationwide collection of databases such as MarketScan include a larger sample size, which provides comprehensive overview of data relevant to the research question throughout the United States. Although retrospective in nature, these databases are based

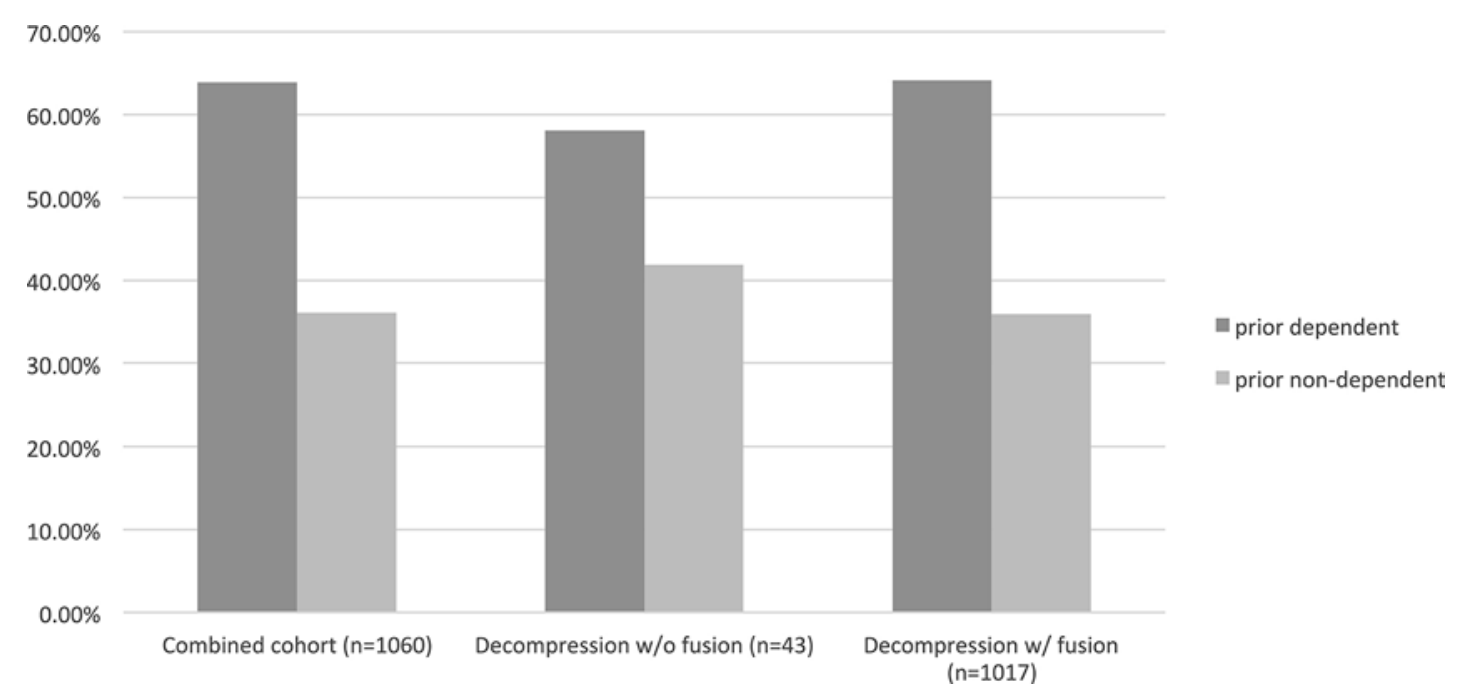

FIG. 5. Bar graph showing the percentage of patients who were opioid dependent after surgery (previously opioid dependent vs not previously opioid dependent) in the combined cohort, in patients who underwent decompression without fusion, and in patients who underwent decompression with fusion for DS. 
TABLE 3. The adjusted effect of patient characteristics on postoperative opioid dependence

\begin{tabular}{lcr}
\hline \multirow{2}{*}{ Variable } & \multicolumn{2}{c}{ Postsurgery Opioid Dependence } \\
\cline { 2 - 3 } & $\mathrm{RR}(95 \% \mathrm{Cl})$ & $\mathrm{p}$ Value \\
\hline Presurgery opioid dependent vs not & $16.29(14.10-18.81)$ & $<0.0001$ \\
\hline Age, 1 -yr increment & $0.972(0.963-0.980)$ & $<0.0001$ \\
\hline Sex: female vs male & $1.166(0.998-1.362)$ & 0.0536 \\
\hline Insurance & & \\
\hline Medicaid vs commercial & $1.337(0.960-1.863)$ & 0.2275 \\
\hline Medicare vs commercial & $1.170(0.929-1.474)$ & 0.9344 \\
\hline Elixhauser score & & \\
\hline 1 vs 0 & $0.956(0.808-1.131)$ & 0.8980 \\
\hline 2 vs 0 & $0.864(0.686-1.088)$ & 0.2038 \\
\hline 3+ vs 0 & $1.045(0.800-1.365)$ & 0.4134 \\
\hline Decompression, w/ vs w/o fusion & $1.038(0.726-1.483)$ & 0.8396 \\
\hline Multilevel vs single-level surgery & $1.107(0.931-1.316)$ & 0.2506 \\
\hline
\end{tabular}

Boldface type indicates statistical significance.

on insurance claims, which mitigate the issue of selection bias while providing a large sample size. MarketScan provides longitudinal information, which includes inpatient and outpatient services, medication refills, and costs.

Apart from our study's inherent limitations of being retrospective, the reliance on data from the MarketScan databases also entails limitations. The insurance claims from which the MarketScan data were acquired were used to bill for in-hospital procedures and outpatient medicine prescriptions, which may not reflect the actual clinical decision-making process used in specific cases or potential confounding variables that affect outcomes. Thus the data may not provide an accurate representation of a problem. The clinical profile of the patients, including additional procedures or clinical diagnosis during the follow-up after the surgery, cannot be determined using this database. Also, claims databases cannot be used to determine the factors that may be associated with opioid dependence such as alcohol/tobacco dependence/abuse and socioeconomic status. However, our study is representative of realworld clinical practice. Potential coding errors and inability to obtain information about the severity and duration of the clinical problem as well as the severity of postoperative complications and quality of life at follow-up are other limitations of such a database. One of the definitions of opioid dependence was based on medication refills prescribed by the physicians for the patients enrolled in the insurance plans. The patients may not have consumed these opioid medications on the refill date, and our analysis may thus overestimate the dependence if there was a delay in actually starting the medications or if they did not consume at all; accordingly, the dependence may have been underestimated in cases in which patients consumed the medications on the same date or beyond the dose prescribed by the physician. Data regarding preoperative and postoperative consumption of morphine equivalents were not included in this database. Also, illegal procurement of opioids is not captured by this database, which may also lead to underestimation of dependence. Nevertheless, our study provides insight into the national trends and factors predictive of opioid dependence following surgical procedures for DS in the United States.

\section{Conclusions}

The majority of patients underwent fusion for DS. Surgical decompression with fusion was associated with decreased postsurgery opioid dependence in patients with DS. Prior opioid dependence is associated with increased risk and increasing age is associated with decreased risk of opioid dependence following surgery for DS. Patients who underwent surgery for DS were twice as likely to become opioid independent as they were to become opioid dependent.

\section{References}

1. Achenbach J, Wagner J, Bernstein L, Johnson J: Trump says opioid crisis is a national emergency, pledges more money and attention. Washington Post. (https:// www.washingtonpost.com/politics/trump-declaresopioid-crisis-is-a-national-emergency-pledgesmore-money-and-attention/2017/08/10/5aaaae327dfe-11e7-83c7-5bd5460f0d7e_story.html?utm_ term=.2af1101945c9) [Accessed February 8, 2018]

2. Adogwa O, Parker SL, Bydon A, Cheng J, McGirt MJ: Comparative effectiveness of minimally invasive versus open transforaminal lumbar interbody fusion: 2-year assessment of narcotic use, return to work, disability, and quality of life. J Spinal Disord Tech 24:479-484, 2011

3. Ambekar S, Sharma M, Kukreja S, Nanda A: Complications and outcomes of surgery for spinal meningioma: a Nationwide Inpatient Sample analysis from 2003 to 2010. Clin Neurol Neurosurg 118:65-68, 2014

4. Armaghani SJ, Lee DS, Bible JE, Archer KR, Shau DN, Kay $\mathrm{H}$, et al: Preoperative opioid use and its association with perioperative opioid demand and postoperative opioid independence in patients undergoing spine surgery. Spine (Phila Pa 1976) 39:E1524-E1530, 2014

5. Brummett CM, Waljee JF, Goesling J, Moser S, Lin P, Englesbe MJ, et al: New persistent opioid use after minor and major surgical procedures in US adults. JAMA Surg 152:e170504, 2017

6. Cepeda MS, Fife D, Ma Q, Ryan PB: Comparison of the risks of opioid abuse or dependence between tapentadol and oxycodone: results from a cohort study. J Pain 14:1227-1241, 2013

7. Connolly J III, Javed Z, Raji MA, Chan W, Kuo YF, Baillargeon J: Predictors of long-term opioid use following lumbar fusion surgery. Spine (Phila Pa 1976) 42:1405-1411, 2017

8. Deyo RA, Gray DT, Kreuter W, Mirza S, Martin BI: United States trends in lumbar fusion surgery for degenerative conditions. Spine (Phila Pa 1976) 30:1441-1447, 2005

9. Elixhauser A, Steiner C, Harris DR, Coffey RM: Comorbidity measures for use with administrative data. Med Care 36:8-27, 1998

10. Finucane TE, Christmas C, Travis K: Tube feeding in patients with advanced dementia: a review of the evidence. JAMA 282:1365-1370, 1999

11. Försth P, Ólafsson G, Carlsson T, Frost A, Borgström F, Fritzell P, et al: A randomized, controlled trial of fusion surgery for lumbar spinal stenosis. N Engl J Med 374:1413-1423, 2016

12. Ghogawala Z, Dziura J, Butler WE, Dai F, Terrin N, Magge $\mathrm{SN}$, et al: Laminectomy plus fusion versus laminectomy 
alone for lumbar spondylolisthesis. N Engl J Med 374:14241434,2016

13. Jacobsen S, Sonne-Holm S, Rovsing H, Monrad H, Gebuhr $\mathrm{P}$ : Degenerative lumbar spondylolisthesis: an epidemiological perspective: the Copenhagen Osteoarthritis Study. Spine (Phila Pa 1976) 32:120-125, 2007

14. Kepler CK, Vaccaro AR, Hilibrand AS, Anderson DG, Rihn JA, Albert TJ, et al: National trends in the use of fusion techniques to treat degenerative spondylolisthesis. Spine (Phila Pa 1976) 39:1584-1589, 2014

15. Littell RC, Stroup WW, Freund RJ: SAS for Linear Models, ed 4. Cary, NC: SAS Institute, 2002

16. Logan J, Liu Y, Paulozzi L, Zhang K, Jones C: Opioid prescribing in emergency departments: the prevalence of potentially inappropriate prescribing and misuse. Med Care 51:646-653, 2013

17. Martin BC, Fan MY, Edlund MJ, Devries A, Braden JB, Sullivan MD: Long-term chronic opioid therapy discontinuation rates from the TROUP study. J Gen Intern Med 26:14501457,2011

18. Mino DE, Munterich JE, Castel LD: Lumbar fusion surgery for degenerative conditions is associated with significant resource and narcotic use 2 years postoperatively in the commercially insured: a medical and pharmacy claims study. J Spine Surg 3:141-148, 2017

19. Norton RP, Bianco K, Klifto C, Errico TJ, Bendo JA: Degenerative spondylolisthesis: an analysis of the Nationwide Inpatient Sample database. Spine (Phila Pa 1976) 40:1219-1227, 2015

20. Phan K, Rao PJ, Kam AC, Mobbs RJ: Minimally invasive versus open transforaminal lumbar interbody fusion for treatment of degenerative lumbar disease: systematic review and meta-analysis. Eur Spine J 24:1017-1030, 2015

21. Quan H, Sundararajan V, Halfon P, Fong A, Burnand B, Luthi JC, et al: Coding algorithms for defining comorbidities in ICD-9-CM and ICD-10 administrative data. Med Care 43:1130-1139, 2005

22. Raebel MA, Newcomer SR, Reifler LM, Boudreau D, Elliott TE, DeBar L, et al: Chronic use of opioid medications before and after bariatric surgery. JAMA 310:1369-1376, 2013

23. Rudd RA, Seth P, David F, Scholl L: Increases in drug and opioid-involved overdose deaths-United States, 2010-2015. MMWR Morb Mortal Wkly Rep 65:1445-1452, 2016
24. Schoenfeld AJ, Nwosu K, Jiang W, Yau AL, Chaudhary MA, Scully RE, et al: Risk factors for prolonged opioid use following spine surgery, and the association with surgical intensity, among opioid-naive patients. J Bone Joint Surg Am 99:1247-1252, 2017

25. Sharma M, Sonig A, Ambekar S, Nanda A: Discharge dispositions, complications, and costs of hospitalization in spinal cord tumor surgery: analysis of data from the United States Nationwide Inpatient Sample, 2003-2010. J Neurosurg Spine 20:125-141, 2014

26. Sharma M, Ugiliweneza, B, Aljuboori, Z, Boakye M. Health care utilization and overall costs based on opioid dependence in patients undergoing surgery for degenerative spondylolisthesis. Neurosurg Focus 44(5):E14, 2018

27. Stokes M, Davis C, Koch G: Categorical Data Analysis Using the SAS System, ed 2. Cary, NC: SAS Institute, 2000

28. Sun EC, Darnall BD, Baker LC, Mackey S: Incidence of and risk factors for chronic opioid use among opioid-naive patients in the postoperative period. JAMA Intern Med 176:1286-1293, 2016

29. Weinstein JN, Lurie JD, Tosteson TD, Hanscom B, Tosteson AN, Blood EA, et al: Surgical versus nonsurgical treatment for lumbar degenerative spondylolisthesis. N Engl J Med 356:2257-2270, 2007

\section{Disclosures}

The authors report no conflict of interest concerning the materials or methods used in this study or the findings specified in this paper.

\section{Author Contributions}

Conception and design: Sharma. Acquisition of data: Ugiliweneza. Analysis and interpretation of data: Sharma. Drafting the article: Sharma. Critically revising the article: Boakye, Sharma, Nuño, Drazin. Reviewed submitted version of manuscript: all authors. Statistical analysis: Ugiliweneza. Administrative/technical/material support: Boakye. Study supervision: Boakye.

\section{Correspondence}

Maxwell Boakye: University of Louisville, School of Medicine, Louisville, KY. maxwell.boakye@ulp.org. 